\title{
EVOLUTION OF PLANETARY NEBULAE ENVELOPES: AN EMPIRICAL APPROACH
}

\author{
V.V. GOLOVATY \\ Astronomical Observatory of Lviv University, 290005 Lomonosov str. 8, Lviv, Ukraine \\ and \\ YU. F. MALKOV \\ Crimean Astrophysical Observatory, 334413 Nauchny, Crimea, Ukraine
}

We carried out the empirical investigation of the evolution of gas density distribution in the envelopes of planetary nebulae (PN). For this purpose we analysed the isophotal maps of $10 \mathrm{PN}$ in the lines $\mathrm{H}$ alpha, $\mathrm{H}$ beta or in the optically thin radio continuum. To obtain the spatial radial distribution of gas density $n(r)$ (where $n=n(H)+n(H e)$ ) we used Abell's integral equation in the simplest, sphericallysymmetric case. We found that $\mathrm{n}(\mathrm{r})$ for all $\mathrm{PN}$ envelopes in our sample can be described by an approximative expression:

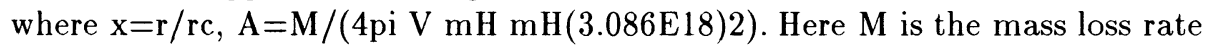
of the star-precursor, $\mathrm{V}$ is the terminal velocity of precursos's wind, $\mathrm{mH}$ is the mass of hydrogen atom; the value of $r c(p c)$ characterizes the age of the envelope. The figure shows the agreement between the formula (1) (dots) and the empirical distributions $n(r)$ (solid curves). The straight line corresponds to precursor's wind with constant $M / V$. It is clearly seen that the formation of the envelope begins from the decrease of precursor's mass loss rate: young PN have the gas density which is less than the density of precursor's wind at the same distance from the center. This conclusion is insensitive to errors in distances to PN (as an example, the dashed curve shows the position of IC 5117 if we increase the distance to this PN by 3 times). Thus, the idea that $P N$ are formed as a result of a rapid increase of precursor's mass loss rate ("superwind") must be ruled out. 